\title{
Evaluating Everyday Competence in Older Adult Couples: Epidemiological Considerations
}

\author{
Roger A. Dixon \\ Department of Psychology, University of Alberta, Edmonton, Alta., Canada
}

\section{Key Words}

Everyday competence, older adults $\cdot$ Epidemiology $\cdot$ Aging couples $\cdot$ Collaborative cognition

\begin{abstract}
Among older adults, everyday competence is often expressed in the context of other participating individuals. Although this active human context may be occasionally comprised of mere acquaintances, long-term partners (such as couples) often act as a unit in engaging in everyday actions or reporting on familiar domains. This special section reflects an important movement in aging research to examine couples as an alternative but normatively common unit of analysis. My discussion focuses on 2 main issues. First, I sketch the rationale, logic, expectation and evidence that long-term couples might develop and display unique advantages in everyday competence. Second, I explore the possibilities that epidemiological principles - thus far applied primarily to individual-level aging, decline and disease - may provide concepts or models for research on long-term changes in couple-level adaptation.
\end{abstract}

Copyright $\odot 2010$ S. Karger AG, Basel

\section{KARGER}

Fax +41613061234

E-Mail karger@karger.ch

www.karger.com
() 2010 S. Karger AG, Basel

Accessible online at:

www.karger.com/ger
As noted by the organizers of this special section [1], the charge presented to the authors of the 4 empirical articles was to report original research inspired by (or pertaining to) the question of 'how does $1+1$ make a couple?' I take this charge to mean: does the mere presence of 2 individuals in close geographical proximity, perhaps engaged in dyadic conversation, constitute a couple? Or must they, in addition, be acquainted, familiar, related, teamed, practiced or in some functional manner interdependent? On the shared ontological and demographic surfaces, the likely answer to this question is apparent: whereas casual dyads are not, couples are indeed partnered (often formally), united in fact or purpose, and linked together over a notable period of time [2]. Accordingly, all of the studies presented in this section include (a) spouses or formally married older adult couples, (b) specific aspects of couple-related attributes (e.g. familiarity, common goals, long-term associations), and (c) various expressions of interrelationships, interactions or joint performances between the partners. Moreover, of the 4 studies, 2 sets of couples $[3,4]$ are investigated while performing activities collaboratively in challenging (and sometimes changing) circumstances, whereas the other 2 sets are examined over time for changes in couple-level attributes $[5,6]$. The study of couples as an alternative but normatively common unit of analysis engaging in every-

Prof. Roger A. Dixon

Department of Psychology

P217 Biological Sciences Building, University of Alberta

Edmonton, AB T6G 2E9 (Canada)

Tel. +1 780492 5850, Fax +1 780492 1768, E-Mail rdixon@ualberta.ca 
day actions or reporting on familiar domains has profound implications for understanding overall competence among older adults. This special section reflects an emerging research emphasis in gerontology and provides an excellent illustration of selected but novel contours of this important direction. In addition, it points to the fact that such research is replete with bewitching possibilities, exasperating complications and promising opportunities.

Therefore, implicit in both the charge posed by the organizers and the new research presented by the contributors were 2 underlying questions, both of which may have far-reaching ramifications in the field of aging. The first is whether the fact of 'couplehood' conveys adaptive advantages, as compared to (a) other forms of overtly equivalent human association (e.g. typical casual dyads) or (b) individual-level performances or outcomes by comparable (biologically, psychologically, socially) older adults. Simple derivative questions may include (a) whether couples perform better in everyday life tasks than do (otherwise equivalent or control) dyads or various instantiations of individuals and (b) whether functioning in a particular domain is systematically related within couples (e.g. as compared with other dyads). The second underlying question is whether any adaptive benefits that might accrue to older couples operate through a set of identifiable and classifiable mechanisms, such as (a) collaborating effectively in solving everyday decision, emotional, practical or cognitive challenges, (b) deriving individuallevel enhancements, support or advantages not routinely available to other aging individuals, or (c) buffering effects through resilience, compensation or accommodation (as a function of uniquely couple-level attributes or interactions).

All the articles in this special section address the backdrop framed by these 2 intriguing questions. In the present commentary I raise 2 general issues for further discussion. The first general discussion issue concerns (a) what it is about 'couplehood' among older adults that may confer everyday interaction and performance advantages and (b) how these advantages might be detected, classified and interpreted. This section contains a brief overview of 2 integral parts of the issue: (a) a rationale for why couples might indeed be measurably advantaged in late-life adaptation, and (b) a perspective on some recent research, including the present articles. For the second general discussion issue, I present an epidemiological view, typically applied to cognitive health and disease with aging, to the question of couples, interaction, collaboration, competence and aging. Specifical- ly, the approach considers the extent to which dynamically changing risk and protection factors, derived from either the individual or couple level of analysis, can affect the trajectories of long-term couples' everyday competence.

\section{The Enduring Rationale: Can Couples Develop and Display Unique Advantages in Everyday Competence?}

\section{Orientation}

In a series of papers [e.g. 7], I frequently used an alliterative and (I hoped) provocative expression: 'Is there compensation through collaboration in cognitive aging?' What I meant to accomplish with such an idiom was to draw attention to the possibility that individual-level cognitive decline, so robustly evident by a bristling accumulation of experimental and longitudinal research in neurocognitive aging, could be buffered in everyday life if older adults recruited and used humans as compensatory cognitive aids. Several supportive but qualified illustrations of this phenomenon have appeared in the literature [e.g. 8, 9]. In this section, I sketch selected definitions, the overall logic and key prevailing theoretical issues. Consistent with the guiding expression - and with a tendency in the literature - the examples used in this article are often drawn from cognition (broadly conceived). Nevertheless, they are expected to apply widely to everyday competence and adaptation in couples.

\section{Definitions and Domains}

Numerous terms are used to refer to 2 critical facts relevant to everyday development and competence. First, other people are an important context of everyday cognitive, social, emotional and even survival activities. Indeed, some of the human social context may be actively or reactively interacting, participating or modulating the activity. Second, some of this interactive or collaborative activity is directed at solving an objectively common task through processes that are often observable and channeled toward a product or outcome that is a function of the interaction. A frequently used term, collaborative cognition, refers to this overall phenomenon, with 3 main provisos. The first qualification is that the term should generously ensure its pre-empirical neutrality with respect to (a) the quality of its dynamic and constituent processes and (b) the adaptive effectiveness of the ensuing and measurable products. A second qualification is that any selected term could be linked to alternative ex- 
pressions referring to overlapping constructs, including cooperative, interactive, transactive, socially shared, conversational and situated cognition [7]. Third, 'cognition' is broadly defined (a) to include a variety of lab-and liferelated processes essential to competent performance in multiple domains of everyday life (including planning, episodic memory, autobiographical memory, problem solving, decision making), and (b) to be associated with other processes (e.g. emotion regulation, well-being, motivation, coping) and conditions (e.g. relationship status, duration and sociocultural considerations) exerting influence on overall adaptation. This special section is about interrelations among dyads, a larger domain that can exist phenomenally and statistically apart from online or in situ target-driven interactions [e.g. 6], but even these interrelations may be associated with underlying or consequent cognitive processes and products [e.g. 3-5]. For example, Berg et al. [3; see also 10] introduced the related term collaborative coping to capture the complementary notion that some of the interactions among spouses in everyday life are directed at solving or coping with nonlaboratory problems of a social-cognitive nature, ranging from resolving everyday hassles to navigating serious stressful life events.

\section{The 'Logic' as Applied to Aging: Conceptual and}

\section{Methodological Challenges}

The processes and products of collaborative life problem solving are not necessarily optimal or even favorable. Appearing in the larger literature are the concepts of process gain and process loss [e.g. 7, 11, 12; see also 1]. Process gain refers to the fact that interacting dyads may operate cooperatively and effectively, leading to or facilitating accurate or adaptive outcomes. Process loss refers to the (unfortunate) corollary that collaborating dyads may also be discoordinated and ineffective. The latter circumstance may promote or permit (a) suboptimal functioning, (b) functioning worse than selected control units or (c) objectively poor functioning. These complicating factors are of particular relevance to research in special cognitive or health populations, where the stakes are theoretically and clinically high. For example, we can imagine the societal benefits if (a) cognitively disadvantaged children could improve their individual approach and performance by participating in collaborative (peer) learning situations, (b) typically aging adults could enhance their global competence or everyday adaptivity through collaborating with friends or colleagues, and (c) select domains of performance by mildly impaired or early Alzheimer's patients could be assisted or supported through collaboration with caregivers or spouses. Despite the apparent fact that a collaborative context envelopes much cognitive, social and emotional activity in the everyday lives of older adults, several theoretical, research and methodological challenges have emerged.

\section{Selected Theoretical and Research Challenges}

The overall research results, comparing processes or performance by various combinations of collaborating groups, have shown some promising effects. However, the coveted uniform patterns in which the mere addition of individuals $(\mathrm{N}+1)$ would function to benefit overall performance either quantitatively or qualitatively [e.g. 8, 13-15] have been elusive. The selectivity of these results may be due to a variety of factors in the epidemiological context of the individuals participating in the group collaborations. Among these are 2 interrelated theoretical complications mentioned in the general literature and in the current set of articles. Specifically, the presence of process loss implies that individual- or dyad-related differences in interactive effectiveness may modulate the overall group's navigation through any given problem. Among these modulators are experience-based differences in the effectiveness of collaborative transactions. These couple-level differences could be attributable to interactive expertise, dyadic familiarity or the degree of related resources for enhanced collaborative processes. For example, interactive expertise may develop with practice and with enhanced knowledge of the partner's strengths and weaknesses [16]. From a complementary perspective, supportive processes such as affordances or attunements may be more readily available to experienced interactants [17]. Similarly, the requirements for cognitive level resources may be reduced when the dyads are highly familiar with one another [4]. In general, a current line of argument may be that compensation through collaboration may occur in cognitive aging, but only when the collaborating units are equipped with some form of interactive expertise [7]. This refinement of the theory should not be disappointing to researchers in interrelations in aging, as a similar refinement is typically imposed at the individual level of analysis; i.e. individual-level cognitive compensation may take many forms, but the probability of it occurring and being effective is enhanced when the individual is experienced in the domain in which it is displayed [e.g. 18].

\section{Methodological Movements}

Several methodological considerations can be derived from the foregoing $[7,13,14]$. First, the dyads (or other 
groups) should be very carefully vetted to ensure that group-level sources of contamination to collaborative expertise (familiarity) are not operating randomly or systematically. These include attention to individual-level differences (within and across groups) in health, personality, neurocognitive integrity, cognitive resources, socioemotional factors, communicative or expressive skills, culture or ethnicity, gender or sensory status. Second, published studies in this field can be enhanced with the presence of well-selected (and equally homogeneous) control conditions, moderating factors and comparison groups. These include individuals, nominal units and age-varying groups [e.g. 4, 8]. Third, the dynamic characteristics of dyads and other groups may be profitably investigated, over long periods longitudinally $[5,6]$, shorter periods with frequent assessments [3] and in investigations of the microprocesses of communication within single sessions [e.g. 4, 19].

\section{Next Steps}

As implied by this commentary and the breadth of the 4 original articles, multiple theoretical and disciplinary strands lead into the study of interrelations among older couples. Because there is no meta-analysis or recent consolidative history of the study of interrelations among older individuals (as dyads or couples) and effects on cognitive and affective adaptation, the present collection provides an excellent window into current research, as well as theoretical and methodological developments [7, $14,20]$. As a next step, it may be possible to integrate, at least at a preliminary level, among the rationales, the disciplinary strands and extant research results - in the direction of articulating complementary approaches to understanding the potential impact and limitations of studying interactions, interrelations and collaborations among older adults (as couples). I turn now to an initial attempt to identify and articulate epidemiological principles that may be useful in moving forward in this field.

\section{Are We Ready for an 'Epidemiology' of Collaborative Competence in Aging?}

The trajectories and outcomes of individual-level cognitive and affective changes with aging are variable and mutiply determined [21]. Pertinent precursors, moderators and mediators originate in levels of analysis as wideranging as biological health, neurobiological, psychological, social-interactional and sociocultural [e.g. 22-24]. Cognition (broadly construed) is typically involved in the achievement of competent actions, even in couples (through the contributions of each individual). The individual trajectories of cognitive aging are dynamic, multidimensional and intraindividually variable. Nevertheless, they are often clustered into several general patterns of change. These include (a) the predominant typical (normal) cognitive aging, which overall tends toward gradual long-term decline, and (b) aging-related accelerations in decline due to distal health comorbidities (e.g., type 2 diabetes, cardiovascular disease), proximal neurological dysfunctions (e.g. stroke, head injury) and especially neurodegenerative disease (such as Alzheimer's disease). Lurking in the background for decades and increasingly evident in longitudinal research [25-27] is a third pattern: (c) trajectories that appear to represent long-term maintenance of competence or selective improvements $[8,9,26]$. Such maintenance may appear in several manifestations, including performance at levels not previously expected, decrements of a lower magnitude than typical, resilience as a function of support (e.g. selection, compensation) and other manifestation of cognitive health with aging. Whichever pattern (or combination) applies, our understanding of general and variable shapes and structures, in the context of multiple precursors and outcomes, has been enhanced by (a) the emergence of large-scale longitudinal or epidemiological studies of aging [28] and (b) the development of methodological tools for evaluating these complex patterns of influence and change [29].

\section{A Bundle of Useful Epidemiological Concepts}

In the health sciences, efforts to characterize and understand a given disease include a well-known collection of epidemiological tasks such as the identification of the causes, distributions, incidence and prevalence rates, as well as risk and protection factors related to them. In gerontology, such information is relevant not only to the study of aging-related diseases (e.g. Alzheimer's disease) but also to normal and healthy aging phenomena [26]. Whatever the outcome, progress can be charted insofar as risk factors (which accelerate decline or hinder maintenance of health) and protection factors (which buffer decline or promote maintenance) can be identified, estimated and tracked. For most complex diseases (including sporadic Alzheimer's disease) risk and protection factors influence health trajectories and outcomes interactively and dynamically - including modifying, supplementing, magnifying or suppressing effects of other precursors. The independent or interactive confluence of factors that lead to cognitive decline (or cognitive health) are usually 
sought in biological, psychological, environmental, lifestyle and social domains. Although useful information can be gathered cross-sectionally, epidemiological studies of developmental phenomena can be optimally framed in time-structured designs. This précis leads to the conjecture that couple-like interrelations among older adults may be studied with epidemiological concepts and approaches.

\section{From a Bundle to a Model: Could Traditional Risk and Protection Factors Apply to Couple-Level Aging?}

There are at least 2 ways. First, couples themselves can be the focus of change, the target of analyses, with their collective attributes and performance the key outcome indicators. Second, in evaluating couple-level aging, each of the constituent individuals, as well as their interactions or interrelations, can be part of the epidemiological context, including operating as risk and protection factors.

\section{Individual-Level Risk and Protection Factors Can \\ Affect Couple-Level Adaptation}

A provisional model representing the changing dynamics of risk and protection factors on the stability of couple-level competence is presented in figure 1. This model applies across a range of domains and would encompass, if not sponsor, studies in which (a) individual members of a couple are evaluated independently or interactively (such as all the interrelations studied in this special section) [6] or (b) the couple itself is the principal unit of analysis (whether or not individual or other dyadic data are collected). As several current authors noted $[3,6]$, individual-level attributes can have an effect on the social-emotional climate of the couple and correspondingly on the competence of the couple's performance in lab or life tasks. The model focuses on the individuallevel attributes of cognitive resources, as these have been extensively studied longitudinally and epidemiologically, and are crucial elements of much everyday competence for couples. The model links temporally the factors potentially influencing (favorably or unfavorably) the concurrent state and longitudinal stability of couple-level adaptation. The middle panel of figure 1 (i.e. the 3 circles) displays the overall goal of maintaining the underlying cognitive reserve necessary for the couple to continue performing everyday tasks at adaptive levels. The concept of dyadic cognitive reserve is invoked informally to reflect the fact that cognitive performance by a couple

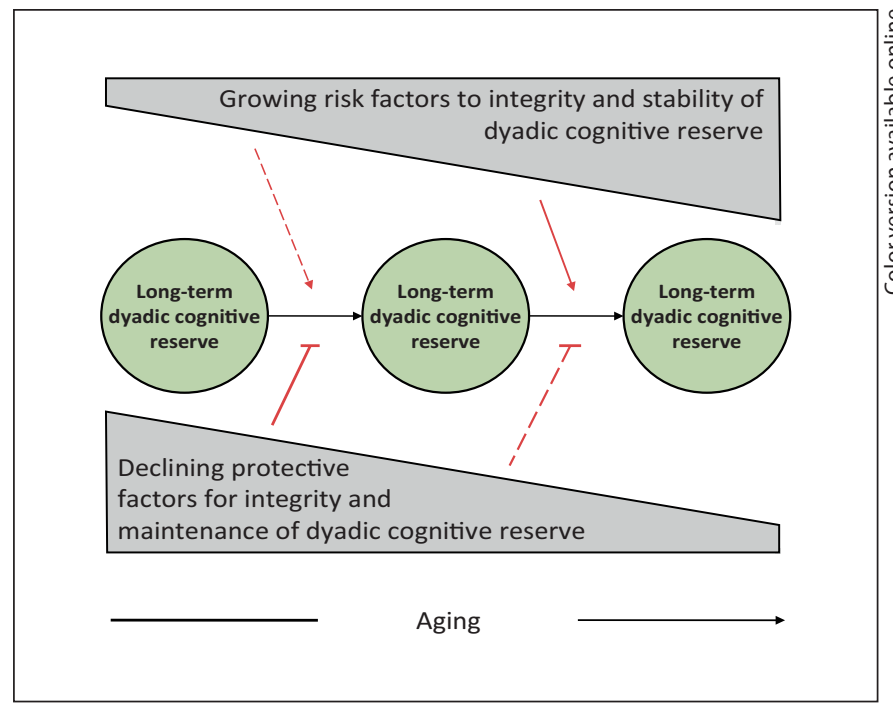

Fig. 1. Differential profiles of risk and protection factors as they influence long-term dyadic cognitive reserve (underlying everyday competence).

is a function of both individuals' ability to contribute to that performance [e.g. 4]. Thus, couple cognitive reserve can be maintained for any given task or domain by (a) both individuals contributing equivalently, (b) both individuals contributing in the typical balance (or asymmetry) to which they have become accustomed, or (c) one individual declining in quantity or quality of contribution but the other dynamically compensating through a variety of potential mechanisms. Hovering like an accumulating ominous cloud over this ideal of long-term couple-level stability, the upper panel of the figure shows the expanding wedge of threats to individual-level cognitive reserve and performance. All of these quickly become risk factors to the integrity and stability of dyadic cognitive reserve. These growing risk factors are essentially the well-known individual-level influences of biological and genetic markers, health and comorbidities, neurodegenerative disease and decline, and many others $[3,21,30,31]$. The declining protective factors are portrayed in the bottom panel as a receding edge (with aging) of individual-level reserve and abilities. Although substantial in earlier parts of adulthood, potential protective factors (e.g. emotional regulation, communication, social engagement, physical activity and fitness, everyday cognitive activity) are at risk for individual-level decline in the extent of support they may provide to any one individual of a dyad [26]. 


\section{Interactional Risk and Protection Factors Unique} to Dyadic Aging May Also Affect Couple-Level

\section{Adaptation}

Another way in which individual-level influences might be crucial elements of couple-level research is implicit but not directly shown in figure 1 . There is a possibility that discoordinated timelines of aging may lead to growing imbalances (or asymmetries) of spousal contributions to dyadic cognitive reserve. Both individuals in a given couple will be aging chronologically at exactly the same rate, but they may very well be aging biologically at quite different rates. This suggests the need for enhanced biological age markers not only in individual-level aging research $[21,30]$ but also in studies of dynamic interrelations in couples. That couples are matched by chronological age does not imply that they are experiencing the same biological aging (i.e. differentially increasing risk factor?) or social-psychological aging (i.e. differentially declining protection factor?). Also important to consider are other modulators of couple-level cognitive stability, such as at least 3 identified by the current authors: (a) quality (not just quantity) of marriage [5], (b) familiarity or interactive expertise [4; see also 7, 16], and (c) attacks on couple-level integrity by acute diseases or accelerated decline in components, or by unprecedented discoordinating changes. Finally, one promising and integrative note should be mentioned. Among the more intriguing exemplars of potential buffering factors for individuallevel normative cognitive decline (and even accelerated transitions into dementia) is a set of factors referred to as social engagement. Briefly, the notion is that social engagement (sustained social activity) can be protective of normal and accelerated cognitive decline due to its stabilizing or supporting role in cognitive practice, cognitive exercise and cognitive reserve [e.g. 27, 32]. Long-term couples may be a paradigm case of the effects of social engagement, as reports derived from ongoing longitudinal studies are already showing. Merging these perspectives may lead to fruitful research.

\section{Conclusion}

The organizers and authors in this special section have targeted and advanced an important emerging research issue in gerontology. Together, they show that couples (a special long-term form of dyads) are a worthy unit of analysis for investigating a wide range of clinically and theoretically relevant aging-related phenomena. Future research may benefit from integrating selected concepts and techniques from corresponding research in aging conducted at the individual level of analysis.

\section{Acknowledgements}

I appreciate support from the National Institute on Aging (R37 AG008235) and the Canada Research Chairs program. I thank Mary Luszcz for her helpful comments on an earlier draft, as well as Jill Friesen and Bonnie Geall for technical support.

\section{References}

1 Hoppmann C, Gerstorf D, Luszcz M: Dyadic interrelations in lifespan development and aging: how does $1+1$ make a couple? - Introduction. Gerontology 2011;57:145-148.

-2 Blieszner R: Introduction: challenges and resilience among later life couples. Generations 2007;31:6-9.

3 Berg CA, Wiebe DJ, Butner J: Affect covariation in marital couples dealing with stressors surrounding prostate cancer. Gerontology 2011;57:168-173.

4 Rauers A, Riediger M, Schmiedek F, Lindenberger U: With a little help from my spouse: does spousal collaboration compensate for the effects of cognitive aging? Gerontology 2011;57:162-167.

5 Strawbridge WJ, Wallhagen MI, Shema SJ: Spousal interrelations in self-reports of cognition in the context of marital problems. Gerontology 2011;57:149-153.
6 Walker R, Luszcz M, Gerstorf D, Hoppmann C: Subjective well-being dynamics in couples from the Australian Longitudinal Study of Aging. Gerontology 2011;57:154-161.

7 Dixon RA: Exploring cognition in interactive situations: the aging of $\mathrm{N}+1$ minds; in Blanchard-Fields F, Hess TM (eds): Social Cognition and Aging. New York, Academic Press, 1999, pp 267-290.

8 Gagnon LM, Dixon RA: Remembering and retelling stories in individual and collaborative contexts. Appl Cogn Psychol 2008;22: 1275-1297.

9 Gerstorf D, Hoppmann CA, Anstey KJ, Luszcz MA: Dynamic links of cognitive functioning among married couples: longitudinal evidence from the Australian Longitudinal Study of Ageing. Psychol Aging 2009;24:296-309.
10 Berg CA, Upchurch R: A developmentalcontextual model of couples coping with chronic illness across the adult life span. Psychol Bull 2007;133:920-954.

11 Hill GW: Group versus individual performance: are $\mathrm{N}+1$ heads better than one? Psychol Bull 1982;91:517-539.

12 Steiner ID: Group Processes and Productivity. New York, Academic Press, 1972.

13 Baltes PB, Staudinger UM (eds): Interactive Minds: Lifespan Perspectives in the Social Foundations of Cognition. New York, Cambridge University Press, 1996.

14 Martin M, Wight M: Dyadic cognition in old age: paradigms, findings, and directions; in Hofer SM, Alwin DF (eds): The Handbook of Cognitive Aging: Interdisciplinary Perspectives. Thousand Oaks, Sage, 2008, pp 629646. 
-15 Ross M, Spencer SJ, Blatz CW, Restorick E: Collaboration reduces frequency of false memories in older and younger adults. Psychol Aging 2008;23:85-92.

16 Engeström Y: Interactive expertise: studies in distributed working intelligence. Univ Helsinki Res Bull 1992, Research Bulletin No. 83.

17 Greeno JG: The situativity of knowing, learning, and research. Am Psychol 1998;53: 5-26.

18 Dixon RA, Bäckman L (eds): Compensating for Psychological Deficits and Declines: Managing Losses and Promoting Gains. Mahwah, Erlbaum, 1995.

19 Gould ON, Dixon RA: Communication during prose recall conversations by young and old adults. Discourse Process 1994;17:149_ 165.

20 Strough J, Margrett J: Overview of the special section on collaborative cognition in later adulthood. Int J Behav Dev 2002;25:2-5.
21 Dixon RA: Enduring theoretical themes in psychological aging: derivations, functions, perspectives, and opportunities; in Schaie KW, Willis SL (eds): Handbook of the Psychology of Aging, ed 7. San Diego, Elsevier, in press.

22 Cabeza R, Nyberg L, Park DC (eds): Linking cognitive and cerebral aging. Oxford, Oxford University Press, 2004.

23 Dixon RA, Bäckman L, Nilsson L-G (eds) New Frontiers in Cognitive Aging. Oxford, Oxford University Press, 2004.

24 Schaie KW, Carstensen L (eds): Social Structures, Aging, and Self-Regulation in the Elderly. New York, Springer, 2006.

25 Baltes PB, Lindenberger U, Staudinger UM: Life span theory in developmental psychology; in Damon W, Lerner RL (eds): Handbook of Child Psychology, ed 6. New York, Wiley, 2006, vol 1: Theoretical Models of Human Development, pp 569-664.

26 Dixon RA: An epidemiological approach to cognitive health in aging; in Bäckman L, Nilsson L-G (eds): Memory, Aging, and the Brain. London, Psychology Press, 2010.
27 Stern Y (ed): Cognitive Reserve. New York, Psychology Press, 2007.

28 Schaie KW, Hofer S: Longitudinal studies in aging research; in Birren JE, Schaie KW (eds): Handbook of the Psychology of Aging, ed 6th ed. San Diego, Academic Press, 2001.

29 McArdle JJ: Latent variable modeling of differences and changes with longitudinal data. Annu Rev Psychol 2009;60:577-605.

30 Anstey K: Cognitive aging and functional biomarkers: what do we know, and where to from here? in Hofer SM, Alwin DF (eds): The Handbook of Cognitive Aging: Interdisciplinary Perspectives. Thousand Oaks, Sage, 2008, pp 327-339.

31 Spiro A, Brady CB: Integrating health into cognitive aging research and theory: quo vadis? in Hofer SM, Alwin DF (eds): The Handbook of Cognitive Aging: Interdisciplinary Perspectives. Thousand Oaks, Sage, 2008, pp 260-282.

32 Fratiglioni L, Wang HX: Brain reserve hypothesis in dementia. J Alzheimer Dis 2007; 12:11-22. 